\title{
Wernicke's encephalopathy in hyperemesis gravidarum
}

\author{
S. Nightingale \\ B.Sc., M.R.C.P. \\ P. D. HEATH
M.Sc., M.R.C.P. \\ P. D. HEATH
M.Sc., M.R.C.P.
}

D. BATES

M.A., M.R.C.P.

S. L. BARRON

M.B., F.R.C.S., F.R.C.O.G.

Departments of Neurology and Obstetrics and Gynaecology, Royal Victoria Infirmary, Newcastle upon Tyne NEI $4 L P$

\section{Summary}

A 20-year-old woman developed severe persistent vomiting early in pregnancy. In three months she lost $18 \mathrm{~kg}$ of body weight: and showed the biochemical features of mild hepatic failure. Four days after starting intravenous dextrose and insulin, she developed short-tsorm memory loss, confusion, ataxia, dysarthria, mild left upper motor neurone facial weakness and upbeat nystagmus. Thiamine was given and no further deterioration occurred. The vomiting persisted until intra-uterine fetal death. Thereafter, her medical, neurological and psychological problems gradually improved. The association of hyperemesis gravidarum, intravenous calorie load and Wernicke's encephalopathy is discussed.

\section{Introduction}

Excessive vomiting in pregnancy sufficiently severe to require hospital admission (hyperemesis gravidarum) is now uncommon and most cases respond to anti-emetic treatment supplemented by parenteral fluid replacement. Neurological complications are rare; we report a patient with refractory hyperemesis in whom a calorie load precipitated Wernicke's encephalopathy.

\section{Case report}

A 20-year-old healthy married woman had her first pregnancy confirmed in December 1980 . Eight weeks after her last menstrual period she developed severe persistent nausea and vomiting. Two weeks later she complained of brief episodes of rotary vertigo, precipitated by certain head movements. The vomiting persisted and in the 20th week of the pregnancy she was admitted to hospital. Her weight had fallen from $82.5 \mathrm{~kg}$ in early pregnancy to $64.5 \mathrm{~kg}$ on admission. Haematological and biochemical investigations were normal, apart from liver function tests-aspartate transaminase $74 \mathrm{u} . / 1$ (normal 4-20); alkaline phosphatase $208 \mathrm{u}$./1 (normal 20-90); bilirubin $42 \mu \mathrm{mol} / 1$ (normal 1.7-17); urinary urobilinogen strongly positive. Ultrasound examination revealed a single fetus compatible with the duration of amenorrhoea.

The vomiting persisted despite anti-emetic therapy, intravenous dextrose and vitamin $\mathrm{K}$ supplements. She developed hyperglycaemia and insulin was added to her regime. Four days after beginning intravenous dextrose therapy her vertigo increased she became ataxic and dysarthric and complained of severe vertical oscillopsia. Parenteral thiamine supplements were started and she was transferred to Newcastle.

Despite almost continuous retching and vomiting and the loss of $18 \mathrm{~kg}$ in the preceding 3 months, she still appeared overweight. She was alert, but on questioning showed evidence of mild confusion, confabulation and a deficit of short-term memory. Pulse was 110 per min in sinus rhythm and blood pressure $110 / 80 \mathrm{mmHg}$. Temperature was $36.5^{\circ} \mathrm{C}$. Cranial nerves were normal, apart from marked upbeat nystagmus in all directions of gaze, an upper motor neurone weakness of the left side of the face and a slurring dysarthria. Examination of the limbs was normal, the deep tendon reflexes were brisk and symmetrical and the plantar responses were flexor. She had a marked truncal ataxia, but no limb ataxia.

The following investigations were normal or negative; full blood count, sedimentation rate, antinuclear factor, autoantibody screen, Australia antigen, serological tests for syphilis, plasma urea, electrolytes and glucose; serum calcium, phosphate, albumin, globulin, and amylase. Liver function tests remained mildly abnormal.

An ultrasound scan demonstrated a viable fetus compatible in size with the duration of the preg- 
nancy. Cranial computerized tomography and cerebrospinal fluid examination were normal.

The patient was given intravenous anti-emetics, multi-vitamin preparations, parenteral feeding and sedation. Her neurological features remained unchanged and the vomiting persisted. At 21 weeks' gestation, the nausea and vomiting improved suddenly and the presence of intra-uterine death was confirmed by a repeat ultrasound scan. In the absence of spontaneous contractions, abortion was induced 4 days later by means of extra-amniotic prostaglandin E2. The fetus and placenta were macroscopically normal.

Thereafter the patient suffered no further vomiting and her neurological and psychological symptoms gradually improved. At 4 months after the onset of hyperemesis gravidarum she was symptom-free and her only signs were of mild upbeat nystagmus and mild truncal ataxia. Her liver function tests returned to normal. She developed a folate deficient megaloblastic anaemia during convalescence which responded to folate supplementation.

\section{Discussion}

Wernicke's encephalopathy is unusual among noninfective neurological disease in that it is both preventable and largely reversible when recognised and treated early (Victor, Adams and Collins, 1971). It is therefore important to anticipate the development of the encephalopathy and to be prepared to treat prophylactically patients who are at risk. Wernicke's encephalopathy may present as an acute or subacute illness with a variety of clinical manifestations; the classical 'Wernicke's triad' of impaired consciousness, ophthalmoplegia and ataxia is neither constant nor frequent (Victor et al., 1971). Wernicke's encephalopathy is probably underdiagnosed; in Harper's large series of necropsies in Perth Australia, Wernicke's encephalopathy was found in $1.7 \%$ of all neuro-pathological necropsies and of these 51 patients, only 7 had been diagnosed during life (Harper, 1979). Although most cases of Wernicke's encephalopathy seen in the Western world today are related to chronic alcoholism, it is vital that physicians recognize other causes of this condition. Wernicke's index case developed the encephalopathy as a result of pyloric stenosis following sulphuric acid ingestion (Wernicke, 1881) and since that time many other non-alcoholic conditions associated with the development of the encephalopathy have been recognized, including severe malnutrition, prolonged vomiting from any cause, thyrotoxicosis and chronic haemodialysis (Dreyfus, 1979). Wernicke's encephalopathy in a patient with hyperemesis gravidarum was first reported by Sheehan in 1939 but there have been few subsequent reports of this association (Campbell and Biggart, 1939; Ebels, 1978).

Our patient, an obese and non-alcoholic woman, suffered severe and refractory hyperemesis gravidarum resulting in gross weight loss, mild hepatic failure and a folate deficient megaloblastic anaemia. After treatment with intravenous dextrose and insulin she developed acutely the neurological and psychological features of the Wernicke-Korsakoff syndrome, with ataxia, vertical nystagmus and a short-term memory deficit. The combination of thiamine deficiency and calorie load is known to be particularly dangerous and may precipitate an acute encephalopathy, occasionally leading to coma, hypothiaminaemic circulatory collapse and sudden death (Victor et al., 1971; Wallis, Willoughby and Baker, 1978). Thiamine supplementation prevented further neurological deterioration but her improvement was minimal until intra-uterine fetal death occurred, resulting in cessation of the hyperemesis. Blass and Gibson (1977) have demonstrated an inherent abnormality of thiamine-dependent enzyme systems in patients with Wernicke's encephalopathy. This may help to explain the relatively small number of alcoholic and vitamin-depleted patients who develop the encephalopathy and also the particular susceptibility to the encephalopathy of some patients, such as the one we report, in the presence of a thiaminedeficient diet.

\section{References}

BLASS, J.P. \& GiBSON, G.E. (1977) Abnormality of a thiaminerequiring enzyme in patients with Wernicke-Korsakoff syndrome. New England Journal of Medicine, 297, 1367.

CAMPBEl, A.C.P. \& BigGaRT, J.H. (1939) Wernicke's encephalopathy (polioencephalitis haemorrhagica superior): its alcoholic and non-alcoholic incidence. Journal of Pathology and Bacteriology, 48, 245.

Dreyfus, P.M. (1979) In: Cecil-Loeb Textbook of Medicine (ed by Beeson, P.B., McDermott, W. \& Wyngaarden, J.B.), p. 712. W. B. Saunders, Philadelphia.

EbELS, E.J. (1978) Letter. Lancet, it, 781.

HARPER, C. (1979) Wernicke's encephalopathy: a more common disease than realized. A neuropathological study of 51 patients. Journal of Neurology, Neurosurgery and Psychiatry, 42, 226.

SheEhAN, H.L. (1939) The pathology of hyperemesis gravidarum and vomiting of late pregnancy. Journal of Obstetrics and Gynaecology of the British Commonwealth, 46, 685.

VICTOR, M., ADAMS, R.D. \& Collins, G.H. (1971) The WernickeKorsakoff Syndrome. Blackwell Scientific Publications, Oxford.

Wallis, W.E., WilloughbY, E. \& BakeR, P. (1978) Coma in the Wernicke-Korsakoff syndrome. Lancet, it, 400.

WERNICKE, C. (1881) Lehrbuck der Gihirnkrankheiten für Ärzte und Studieren, Vol. 2, p. 229. Theodor Fischer, Kassel. 